lignocaine can curtail chronic migraine and analgesic rebound headache (1). Further, ketamine provides short-term analgesia and enables reduction in central sensitisation of pain pathways, particularly in the setting of codeine/opiod overuse (2). This paper describes use of subcutaneous lignocaine and ketamine infusion in chronic migraine.

Methods A prospective observational cohort study was undertaken in patients with chronic migraine. Patients received a prolonged subcutaneous lignocaine and ketamine infusion (mean duration 11 days) and underwent evaluation at fourtime points over six months. The effects on the excitability of motor axons in the median nerve were documented using standard procedures.

Results Fourteen patients were recruited. The infusion was well tolerated; no major side effects were seen. There were no significant long-term changes in the excitability of motor axons. At six months, 13/14 patients had sustained benefit. Three of 4 patients remained free of analgesic rebound headache. One patient remained headache-free. Conversion to episodic migraine occurred in 6/14. Improvement in chronic migraine was reported by $6 / 14$. Three of six were able to return to work, with 1 returning to studies. Benefit was greater in those with depression and history of opiod/codeine use.

Conclusion Subcutaneous lignocaine and ketamine can help break entrenchment in chronic migraine as part of a structured management plan.

\section{THE EFFECT OF CLADRIBINE TABLETS ON DELAYING THE TIME TO CONVERSION TO CLINICALLY DEFINITE MULTIPLE SCLEROSIS (MS) OR MCDONALD MS IS CONSISTENT ACROSS SUBGROUPS IN THE ORACLE-MS STUDY}

\begin{abstract}
${ }^{1}$ James Bowen, ${ }^{2}$ Alan Gillett ${ }^{*},{ }^{3}$ Doris Damian, ${ }^{3}$ Yann Hyvert, ${ }^{3}$ Fernando Dangond, ${ }^{4}$ Megan Grosso, ${ }^{5}$ Thomas Leist. ${ }^{1} M S$ Center at the Swedish Neuroscience Institute, Seattle, WA, USA; ${ }^{2}$ EMD Serono Inc, Mississauga, ON, Canada; ${ }^{3} E M D$ Serono, Inc, Billerica, MA, USA; ${ }^{4}$ EMD Serono, Inc., Rockland, MA, USA; ${ }^{5}$ Division of Clinical Neuroimmunology, Thomas Jefferson University, Jefferson Medical College, Philadelphia, PA, USA
\end{abstract}

\subsection{6/jnnp-2019-anzan.78}

Introduction In the Phase 3 ORACLE-MS trial in 616 subjects with a first demyelinating event at high risk of converting to multiple sclerosis (MS), treatment with cladribine tablets 10 $\mathrm{mg}(3.5 \mathrm{mg} / \mathrm{kg}$ or $5.25 \mathrm{mg} / \mathrm{kg}$ cumulative dose over 2 years [CT3.5 and CT5.25, respectively]) significantly delayed time to conversion to clinically definite multiple sclerosis (CDMS) according to Poser criteria $(67 \%$ or $62 \%$ risk reduction [RR], respectively) and time to conversion to $2005 \mathrm{McD}$ onald MS (50\% or $57 \%$ RR, respectively), versus placebo. The objective was to analyze the effect of cladribine tablets vs placebo on converstion to CDMS and McDonald MS across ORACLE-MS patient subgroups based on baseline characteristics.

Methods In this post-hoc analysis, time-to-conversion to CDMS or McDonald MS over the double-blind period was analyzed for patients treated with CT5.25 ( $=204)$, CT3.5 $(\mathrm{N}=206)$ or placebo $(\mathrm{N}=206)$ across different subgroups. Subgroups were defined by baseline characteristics which have been investigated as potential predictors of CDMS conversion (age $[<30$ or $\geq 30$ years], gender, first classification demyelinating event [monofocal or multifocal], presence of T1 Gd+ lesions and number of T2 lesions $[<9$ or $\geq 9]$ ).

Results Treatment with CT3.5 or CT5.25 was consistently efficacious across the subgroups examined on conversion to
CDMS versus placebo for most comparisons (RR range: CT3.5, 39\%-72\%; CT5.25, 36\%-79\%). Similarly, treatment effect of both doses on conversion to 2005 McDonald MS was consistent across subgroups (CT3.5,40\%-59\%; CT5.25,42\%-79\%).

Conclusions The effect of cladribine tablets on delaying the time-to-conversion to CDMS, or to McDonald MS, is consistent across subgroups.

\section{CEREBELLAR OEDEMA IN FULMINANT ADULT LEIGH SYNDROME}

${ }^{1}$ Leon S Edwards*, ${ }^{2}$ Gabor M Halmagyi, ${ }^{2,3}$ Michael H Barnett, ${ }^{1}$ Christopher A Blair,

${ }^{2,3}$ Matthew C Kiernan. ${ }^{1}$ Neurology, Liverpool Hospital, Liverpool, NSW, Australia; ${ }^{2}$ Institute of Clinical Neuroscience, Royal Prince Alfred Hospital, Camperdown, NSW, Australia; ${ }^{3}$ Brain and Mind Centre, University of Sydney, Camperdown, NSW, Australia

\subsection{6/jnnp-2019-anzan.79}

Introduction We report a case of adult Leigh syndrome resulting in rapidly fatal cerebellar oedema.

Case A 19-year-old female presented with a five-week history of hyperventilation, generalised weakness, dysarthria and bilateral ptosis. Brain Magnetic resonance imaging (MRI) findings and the presence of a mitochondrial mutation (NC_012920.1 (MT-ATP6):m.9176T $>$ C) in blood and urine with approximately $97 \%$ heteroplasmy, confirmed a diagnosis of Leigh syndrome.

Two-days after a normal lumbar puncture, opening pressure $8 \mathrm{~cm}$ water, her conscious level rapidly declined. CT revealed marked cerebellar oedema with brainstem compression. Despite immediate decompression, she did not recover consciousness and died six-weeks after symptom onset.

Conclusion Adult Leigh syndrome is a progressive untreatable inherited mitochondrial disorder typically of infants and children. Adult cases are rare and described mostly in single case reports. There is marked phenotypic and genotypic variability. Over $83 \%$ of Leigh's syndrome is identified by the age of 2 years, however, there have been cases reported in patients up to 74 years old. There are over 60 mutations described in Leigh syndrome, which are identified in only half of reported cases. Classic MRI changes include bilateral symmetric T2 hyper-intensities in the basal ganglia and brainstem. To our knowledge, this is the first reported case resulting in fulminant cerebellar oedema. A challenge of diagnosis remains the marked heterogeneity in presenting symptoms including cognitive decline, behavioural change and ophthalmoparesis. Typically, this syndrome has been confirmed by histopathology at autopsy. Advances in genetics and imaging have allowed earlier accurate diagnosis, potentially paving the way for improved therapeutics.

\section{MOTOR NEURON DISEASE WITH MALIGNANCY: CLINICAL AND PATHOPHYSIOLOGICAL INSIGHTS}

${ }^{1}$ Mana Higashihara*, ${ }^{2}$ Parvathi Menon, ${ }^{2}$ Nimeshan Geevasinga, ${ }^{1}$ Mehdi AJVan den Bos, ${ }^{3}$ Matthew C Kiernan, '2Steve Vucic. 'Neurology, Westmead Hospital, Westmead, NSW, Australia; ${ }^{2}$ Neurology, Westmead Clinical School, University of Sydney, Westmead, NSW, Australia; ${ }^{3}$ Brain and Mind Centre, University of Sydney, Sydney, NSW, Australia

\subsection{6/jnnp-2019-anzan.80}

Introduction While some regard an association between motor neuron disease (MND) and malignancy as co-incidental, others 
have argued that it could represent a distinct clinical entity. The present study undertook in depth phenotyping along with assessment of cortical function to further explore disease pathophysiology in MND with malignancy (MND-M) patients.

Methods Clinical features along with assessment of peripheral and cortical function was undertaken in 13 MND-M and results were compared to sporadic and familial MND cohorts. Results From a cohort 13 patients (10 males; aged 65.2 \pm 2.0 years), $30.8 \%$ were diagnosed with a haematological malignancy. The lower motor neuron phenotype predominated in the in the MND-M patients $(\chi 2=10.8, P<0.01)$, with the upper motor neuron (UMN) score being significantly reduced in MND-M patients compared to sporadic and familial MND cohorts $(\chi 2=6.84, \mathrm{P}<0.01)$. The neurological deficits did not respond to treatment of the underlying malignancy in the majority of MND-M (92\%) patients, and as such there were no significant differences in survival between the cohorts. Despite a paucity of UMN signs, cortical hyperexcitability was evident in MND-M patients, as indicated by reduction in short interval intracortical inhibition $(\mathrm{P}<0.01)$ and increase in motor evoked potential amplitude $(\mathrm{P}<0.01)$, that were similar to findings in sporadic and familial MND cohorts.

Conclusions The present study suggests that MND-M falls within the spectrum of MND. A co-incidental association between MND and malignancy is underscored by cortical dysfunction and clinical findings which seems within the spectrum of abnormality evident in classical MND phenotypes.

\section{HOW TO DIAGNOSE LEWY BODY DEMENTIA? PREVALENCE AND UNDERLYING RELATIONSHIP BETWEEN CLINICAL AND NEUROPSYCHOLOGICAL FEATURES OF DLB}

Elie Matar*, Kaylena A Ehgoetz Martens, Glenda M Halliday, Simon JG Lewis. Brain and Mind Centre, University of Sydney, Camperdown, NSW, Australia

\subsection{6/jnnp-2019-anzan.81}

Introduction Despite its importance for management, prognostication and selection of patients for clinical trials, the diagnosis of Dementia with Lewy Bodies (DLB) remains challenging. Complicating this is a recent change in the diagnostic criteria which has arguably shifted the expected phenotype of DLB patients. In this study we aimed to characterize and examine the relationship between cognitive and clinical diagnostic variables in DLB patients to uncover latent symptom clusters that may streamline future diagnostic approaches in the clinic.

Methods The clinical and neuropsychological profile of 27 prospectively recruited participants diagnosed with probable DLB and 25 age-matched controls was characterized according to the most recent consensus criteria.Symptoms were scored using a novel combination of established clinical and research instruments.

Results We demonstrate comparable sensitivity of formal neuropsychological testing and bedside screening tools (MOCA/ MMSE) for identifying domain-specific differences between controls and patients $(\mathrm{p}<0.001)$. Optimal sensitivity thresholds for diagnosis of Parkinsonism (88.9\%) were explored yielding a prevalence range of 50\%-90\% within our cohort.Factor analysis using all core and supportive features of the diagnostic criteria identified 6 independent factors accounting for $81 \%$ of the total variance. Unique relationships identified included between hallucinations and fluctuations and excessive daytime somnolence; between REM sleep behavior disorder and orthostatic hypotension; and Parkinsonism and urinary disturbance. 'Prodromal' symptoms including autonomic and early neuropsychiatric features are represented in the remaining factors.

Conclusion Parsimonious delineation of clinical variables using identified symptom clusters can aid DLB diagnosis.Clusters are also used to highlight latent pathological relationships. Appropriate instruments and thresholds for detecting dementia and core and suggestive features are presented.

\section{TOWARDS OBJECTIVE TESTING IN PARKINSON'S DISEASE: A SYSTEMATIC REVIEW OF THE LITERATURE LOOKING AT ASSESSMENT OF POSTURAL SWAY}

${ }^{1}$ Wenbo Ge*, 2,1 Deborah Apthorp, ${ }^{3,4}$ Christian J Lueck, ${ }^{1,5,6,7}$ Hanna Suominen. 'Research School of Computer Science, The Australian National University, Canberra, ACT, Australia; ${ }^{2}$ School of Psychology and Behavioural Sciences, University of New England, Armidale, NSW, Australia; ${ }^{3}$ Department of Neurology, Canberra Hospital, Canberra, ACT, Australia; ${ }^{4}$ Medical School, The Australian National University, Canberra, ACT, Australia; ${ }^{5}$ Machine Learning Research Group, Data61/CSIRO, The Australian National University, Canberra, ACT, Australia; ${ }^{6}$ Faculty of Science and Technology, University of Canberra, Bruce, ACT, Australia; ${ }^{7}$ Department of Future Technologies, University of Turku, Turku, Finland

\subsection{6/jnnp-2019-anzan.82}

Introduction Parkinson's Disease (PD) is associated with increased mortality and reduced quality of life. There is currently no accurate objective measure for use in diagnosis or assessment of severity. Analysis of postural sway may help in this regard. This systematic review aimed to assess the effectiveness of the various features currently used to analyse postural sway.

Methods Five databases were searched for articles that examined postural sway in both PD patients and controls. An effect size (ES) was derived for every feature reported in each article. The most effective features and feature-families were determined, along with the influence on these measures of data sampling rate and experimental condition.

Results 441 papers were initially retrieved, of which 31 met the requirements for analysis. The most commonly-used features were not the most effective (e.g. PathLength had an ES of 0.47 while TotalEnergy had an ES of 1.78). Decreased sampling rate was associated with decreased ES (e.g. ES of PathLength lowered from 1.12 at $100 \mathrm{~Hz}$ to 0.40 at $10 \mathrm{~Hz}$ ). Being off medication was associated with a larger ES (e.g. ES of PathLength was 0.21 on medication and 0.83 off medication).

Conclusions Some measures of postural sway are better able to distinguish PD patients from controls than others. ES is enhanced by using a higher sampling rate and studying patients off medication. These results will inform future studies looking at postural sway in PD and contribute to the aim of finding an objective marker of the disease.

\section{PREDICTING PARKINSON'S AND DEMENTIA WITH LEWY BODIES (PRE-D) RESEARCH STUDY - A SYDNEY-BASED LONGITUDINAL BIOBANKING PROGRAM}

${ }^{1}$ Elie Matar*, ${ }^{1}$ Kaylena A Ehgoetz Martens, ${ }^{2}$ Maria Comas Soberats, ${ }^{1}$ Glenda M Halliday, ${ }^{1}$ Simon JG Lewis. ${ }^{1}$ Brain and Mind Centre, University of Sydney, Camperdown, NSW, Australia; ${ }^{2}$ Woolcock Institute of Medical Research, Sydney, NSW, Australia

\subsection{6/jnnp-2019-anzan.83}

Introduction Idiopathic REM sleep behaviour(iRBD) disorder represents the most specific prodromal marker of an 